43-44 | 2013

Le pastoralisme en Haute-Asie : la raison nomade dans l'étau des modernisations

Fauconnet-Buzelin Françoise, Les martyrs oubliés du Tibet. Chronique d'une rencontre manquée (1855-1940) Paris, Les éditions du cerf, 2012. ISBN 978-2-204-09593-8

\title{
Katia Buffetrille
}

\section{OpenEdition Journals}

Édition électronique

URL : https://journals.openedition.org/emscat/2187

DOI : $10.4000 /$ emscat.2187

ISSN : 2101-0013

Éditeur

Centre d'Etudes Mongoles \& Sibériennes / École Pratique des Hautes Études

\section{Référence électronique}

Katia Buffetrille, «Fauconnet-Buzelin Françoise, Les martyrs oubliés du Tibet. Chronique d'une rencontre manquée (1855-1940) », Études mongoles et sibériennes, centrasiatiques et tibétaines [En ligne], 43-44 |

2013, mis en ligne le 20 septembre 2013, consulté le 13 juillet 2021. URL : http://

journals.openedition.org/emscat/2187 ; DOI : https://doi.org/10.4000/emscat.2187

Ce document a été généré automatiquement le 13 juillet 2021.

(c) Tous droits réservés 


\title{
Fauconnet-Buzelin Françoise, Les martyrs oubliés du Tibet. Chronique d'une rencontre manquée (1855-1940)
}

Paris, Les éditions du cerf, 2012. ISBN 978-2-204-09593-8

\author{
Katia Buffetrille
}

\section{RÉFÉRENCE}

Fauconnet-Buzelin Françoise, Les martyrs oubliés du Tibet. Chronique d'une rencontre manquée (1855-1940), Paris, Les éditions du cerf, 2012. ISBN 978-2-204-09593-8

1 Tout anthropologue travaillant dans les marches tibétaines, aux confins du Sichuan et du Yunnan, sait que la correspondance des missionnaires des Missions Étrangères de Paris peut être une source d'information fort utile, en dépit de l'étroitesse d'esprit de la plupart d'entre eux.

2 Françoise Fauconnet-Buzelin, chargée de recherche aux Missions étrangères de Paris, s'intéresse dans ce livre à huit missionnaires qui ont été assassinés dans les marches tibétaines afin de déterminer s'ils peuvent être qualifiés de martyrs ou non.

3 Le titre du livre de Françoise Fauconnet-Buzelin met bien en avant les deux objectifs de cet épais volume (654 pages) mais si le titre répond à la question posée, à la lecture, le texte ne donne pas une réponse aussi définitive. En revanche, l'auteur montre bien que la rencontre entre les propagateurs du catholicisme et les religieux tibétains a été un échec total.

4 Comme l'indique l'auteur dans son introduction, ce livre est écrit dans une perspective «non pas purement historique, mais religieuse et missiologique, et si possible spirituelle » (pp. 16-17).

5 L'auteur raconte la vie de ces missionnaires, de leur enfance à leur engagement, puis leur arrivée à leur poste et leurs incessants déplacements au gré des troubles dont la 
description finit par semer la confusion dans l'esprit du lecteur. Ces hommes avaient de nombreux points communs : des jeunes gens dont la foi confine à l'exaltation décident de consacrer leur vie à la propagation de la parole du Christ auprès des populations "idolâtres ", prêts dans leurs débuts à mourir en martyr pour prouver leur foi, une résolution qui s'estompe à mesure que les années passent au point que les missionnaires n'hésitent pas à prendre les armes pour se défendre. Aucun doute ne les effleure quant à la légitimité de leur action. Leur mépris pour la culture et la civilisation tibétaines ne pouvaient effectivement que leur attirer des inimitiés de la part des religieux et aussi des populations locales. Les rares missionnaires qui montraient un intérêt pour autre chose que la propagation de la foi, étaient décrits, tel le Père Goré, comme " pas trop excitateur de piété parmi ses chrétiens, trop large sur les libertés que peut prendre un missionnaire, va assister aux danses et aux comédies des lamas, comme les ministres protestants, et trouvant au moins étrange qu'on ne le permette pas ", selon les termes du vicaire apostolique (p. 576). Il est vrai que les missionnaires protestants, comme le Père Shelton (il est dommage que l'auteur n'ait pas lu le livre qui lui a été consacré par D. A. Wissing, Pioneer in Tibet. The life and Perils of Dr Albert Shelton, 2004, New York: Palgrave Macmillan) qui passa plus de 20 ans dans la même région et à la même époque, ont fait montre d'une ouverture d'esprit et d'un intérêt pour la population qui allait bien au-delà de la simple conversion.

L'auteur a une grande connaissance de la littérature missiologique mais on peut regretter son manque de connaissance sur le Tibet en général et parfois, la reprise à son compte des préjugés des missionnaires. Ainsi, l'utilisation du terme lamaserie au lieu de monastère qui propage l'idée que tous les moines tibétains sont des lamas. Au Tibet, tous les religieux ne sont pas des moines, et si la population monastique était traditionnellement très importante, le nombre de lamas proprement dits l'était beaucoup moins. Un lama (sanskrit guru) est un maître spirituel, détenteur d'une lignée de transmission tantrique et qui, à ce titre, peut donner les enseignements et initiations de la voie des tantra. Il est certain que, comme le précise l'auteur (p. 263), que les missionnaires avaient une vision caricaturale du bouddhisme tibétain qu'ils voyaient plus comme une doctrine satanique que comme une religion. Elle en donne de nombreux exemples. Il est vrai que de nombreux moines ne menaient pas la vie qu'un Occidental estime devoir être celle d'un religieux, mais le système du monachisme tibétain n'étant en rien comparable à celui du catholicisme, il aurait fallu donner au lecteur plus de clés pour le comprendre. Le Tibet a une civilisation complexe et très riche. Certes, il existait un système de corvées lourdes et inégalitaires et des taxes nombreuses. Par ailleurs, une forme d'esclavage (dont ont profité grandement les missionnaires) était couramment pratiquée durant la première moitié $\mathrm{du} \mathrm{xx}^{\mathrm{e}}$ siècle dans les régions limitrophes orientales et méridionales qui consistait essentiellement pour les Tibétains à maintenir en esclavage des membres des tribus voisines. La connaissance exclusive du Tibet à travers les écrits des missionnaires conduit l'auteur à écrire que «les prêtres français pouvaient à juste titre se prévaloir d'une action civilisatrice supérieure à la féodalité oppressive imposée au peuple par le clergé tibétain » (p. 639). Or le Tibet n'était pas qu' « une féodalité oppressive » et l'idée d'une action «civilisatrice supérieure » teintée de colonialisme des missionnaires reste des plus douteuses.

7 Il est regrettable que l'auteur ne se soit pas renseigné auprès de tibétologues pour une bibliographie correcte sur la culture et la religion du Tibet. Cela la conduit à s'appuyer sur des auteurs comme F. Lenoir et L. Deshayes lorsqu'elle parle du mouvement rimé (ris 
med) et de l'école gelugpa que Lenoir-Deshayes qualifient de lignée. S'il est vrai que ce mouvement a en partie été élaboré en réaction contre certain Gelugpa, d'autres y ont contribué. Donner un jugement aussi négatif sur l'ensemble des Gelugpa du XIXe siècle montre bien l'ignorance des auteurs. Les Gelugpa ont pris les armes pour défendre leurs institutions face aux troupes chinoises dans le Khams à la fin du XIX et au début $\mathrm{du} \mathrm{xx}^{\mathrm{e}}$ siècles... Les missionnaires chrétiens ont été simplement des victimes collatérales de ce conflit, car vus par les Tibétains comme des alliés des Chinois sans titres de séjour valides (ils devaient leur présence à des permis chinois...) Il y aurait beaucoup d'autres choses à dire sur ce court passage mais ce compte-rendu n'en est pas le lieu.

8 La société tibétaine était certes très hiérarchisée, confinée dans un mode de vie traditionnel, inégalitaire et une grande partie de la population vivait d'une production de subsistance. Seuls l'État, le clergé et les nobles pouvaient être propriétaires. Les paysans, la grande majorité de la population, étaient héréditairement liés à la terre et assujettis à des taxes et corvées qui frappaient non l'individu mais la maisonnée. Elles n'excluaient donc nullement les déplacements d'une partie des membres de la maisonnée. Par ailleurs, un système légal existait auquel les paysans pouvaient faire appel. La vie était difficile mais à l'égale de celle de nombreuses sociétés traditionnelles. Il convient de noter qu'en dehors de catastrophes naturelles, le Tibet n'a connu ni famine, ni soulèvement populaire notable.

9 Cet ouvrage s'adresse à un lectorat particulier que la vie des missionnaires dans ces contrées lointaines intéresse. Le scientifique pourra éventuellement y découvrir un missionnaire qui a laissé une abondante correspondance sur une région particulière. Mais on peut regretter l'absence d'une "voix » tibétaine afin de faire contrepoids à celles des missionnaires. 